\title{
Complejidad de la estructura espacio-temporal de la precipitación
}

\author{
Óscar José Mesa-Sánchez, Victor Manuel Peñaranda-Vélez* \\ Departamento de Geociencias y Medio Ambiente, Universidad Nacional de Colombia, Sede Medellín, Colombia.
}

\begin{abstract}
Resumen
El entendimiento de los procesos de precipitación tiene implicaciones prácticas importantes que comprenden el dimensionamiento de obras de evacuación de aguas lluvias, la prevención y atención de desastres, la planificación de la ocupación territorial, el planeamiento y operación de recursos hídricos y el funcionamiento de los ecosistemas naturales, agropecuarios y urbanos. Sin embargo, su irregularidad no ha sido descifrada todavía. Existen diversos desarrollos matemáticos para tratar de describir la dinámica espacio - temporal de este complejo proceso hidrológico, pero todavía las predicciones espacio - temporales no son aceptables. En los primeros trabajos que se reportan en la literatura científica hubo interés por estudiar la estructura espacio - temporal de la precipitación mediante análisis estadísticos para caracterizar la variabilidad o aleatoriedad de sus observaciones. Pero aún en tal terreno existen limitaciones para una descripción completa de la estructura estocástica de los campos de precipitación, los modelos tradicionales no han resultado apropiados, su estructura es muy suave para una adecuada caracterización de un campo muy irregular, y la alternativa exige proliferación de parámetros y de hipótesis, lo que no es satisfactorio. Además de los retos de encontrar descripciones adecuadas, se ha vuelto crucial incorporar la dinámica del proceso físico, lo que puede venir de una integración de la termodinámica con la dinámica atmosférica y la turbulencia, para así avanzar en la predicción. Este artículo de revisión describe las principales características observadas de la precipitación, los problemas más notables en el intento por su explicación y los retos derivados de su complejidad.
\end{abstract}

Palabras clave: precipitación, aleatoriedad, multifractales, ciencias hidrológicas.

Complexity of the space-time structure of rainfall

\begin{abstract}
Understanding precipitation processes has important practical implications comprising the dimensioning of rainwater evacuation structures, disaster prevention planning, territorial occupation planning, water resources management and the performance of natural, agricultural and urban ecosystems. However, its inherent irregularity has not been deciphered. There are various mathematical developments attempting to describe the space - time dynamics of this complex hydrological process, but they are not adequate enough. Among the former works reported in the scientific literature, the study of the space - time structure of rainfall was rendered by mean of statistical analyzes in order to characterize the variability and randomness of their observations. But even in this field there are limitations for a complete description of the stochastic structure of rainfall fields, traditional models have not been appropriated, they produce smooth functions to characterize a very irregular field and the improvement of these models requires the proliferation of parameters and hypothesis, which is not satisfactory. In addition to the challenges of finding adequate descriptions, it has become crucial to incorporate the dynamics of the physical process, which should come from an integration of thermodynamics, atmospheric dynamics and turbulence, enabling some progress in prediction. This review paper describes the main features of the space - time structure of the precipitation fields, points out the difficulties for its understanding and explores the challenges coming from its complexity.
\end{abstract}

Key words: Precipitation, rainfall, randomness, multifractals and hydrological sciences.

\section{Introducción}

El aumento de la población y el desarrollo económico han incrementado rápidamente la demanda de agua, lo que hace más urgente comprender los patrones del comportamiento del recurso hídrico y predecir su dinámica ante escenarios futuros. La precipitación como una de las componentes fundamentales del ciclo hidrológico es además determinante del sistema climático global, participa en la dinámica y en la composición atmosférica, por un lado por el papel directo del agua en sus diferentes fases, por sus importantes

\footnotetext{
*Correspondencia:

Victor Manuel Peñaranda Vélez, vmpenarandav@unal.edu.co

Recibido: 17 de febrero de 2015
}

Aceptado: 15 de mayo de 2015 
propiedades ópticas y energéticas, asociadas a la liberación de calor latente, a los cambios de presión por cambio de fase y al efecto invernadero del vapor de agua. Por otro lado, de manera indirecta también afecta el transporte y remoción de aerosoles y gases solubles (Mesa, 2007). Dada su complejidad intrínseca, la precipitación debe ser estudiada desde un amplio marco temático para responder a los intereses de sostenibilidad social y económica de todas las regiones del planeta.

Como puede evidenciarse en el último reporte presentado por el Panel Intergubernamental del Cambio Climático, desde 1951 se presenta un incremento en la frecuencia y en la intensidad de las fuertes precipitaciones sobre varias regiones del planeta (IPCC, 2013; p.2-162); así mismo, sobre la región tropical $\left(30^{\circ} \mathrm{S}-30^{\circ} \mathrm{N}\right)$, se identifican tendencias positivas en las anomalías de la precipitación anual desde mediados del año 1990 (IPCC, 2013; p.2-162). Otros estudios desarrollados, como el reportado por Mesa, Poveda \& Carvajal (1997) para Colombia, encuentran tendencias positivas en las fases del ciclo anual y semianual de los registros de precipitación de 151 estaciones distribuidas sobre el país; estos y otros resultados han sido entendidos como la intensificación de eventos hidrológicos extremos que se traducen en pérdidas humanas y económicas. Apoyado en las sugerencias de Mesa, et al. (1997), en Colombia se debe actuar diligentemente en el desarrollo de estudios climáticos que logren anticiparse a las consecuencias negativas de los procesos hidrometeorológicos, por los beneficios no solo para el campo científico sino también en la sociedad. Por ejemplo, toda la metodología del diseño hidrológico en ingeniería se apoya en la hipótesis de estacionariedad. En consecuencia se asume que el registro histórico de mediciones en un sitio dado se puede usar para inferir estadísticamente el comportamiento futuro, de donde se deducen los requerimientos de diseño para una estructura que está en proceso de planeación y que va a operar por varias décadas. La falla en la hipótesis de estacionariedad obliga a reconsiderar todas las metodologías de diseño. En principio no es posible un ajuste simple, porque las actuales metodologías no están fundadas en un conocimiento científico sino empírico.

Una demostración clara de la necesidad de avanzar en el entendimiento de la precipitación es la ausencia de una teoría que permita predecir el efecto del calentamiento global en la precipitación. Hay estudios estadísticos de las tendencias recientes, hay modelos globales y hay elementos que permiten afirmar que uno de los efectos será la aceleración del ciclo hidrológico. Pero la respuesta a dónde, cuánto y cuándo van a ser los cambios, tiene bastante incertidumbre, a pesar de la enorme importancia práctica de estas preguntas.

Este artículo centra su visión en los aspectos que caracterizan a la precipitación como un proceso geofísico altamente complejo. El objetivo es hacer una revisión crítica de los principales elementos conceptuales que han sido fundamentales para la descripción de la precipitación y prospectivamente discutir una ruta para avanzar en la explicación de la inherente complejidad de este proceso geofísico.

El presente artículo se estructura en cinco secciones. En esta primera sección se presenta una introducción general al tópico de estudio, el cual ilustra la importancia de estudiar la precipitación no sólo desde un contexto puramente científico, sino también abordando el contexto social en el cual la precipitación juega un papel substancial para el desarrollo de la sociedad. En la Sección 2 se presentan tres aspectos vitales identificados por el Consejo Nacional de Investigación Americano (National Research Council, 1991) que deben tenerse en cuenta para la descripción de la precipitación: la escala en que se desarrolla, la alta variabilidad de los patrones observados y la estructura conceptual de los modelos usados para representar estos patrones. La Sección 3 se orienta a dar una idea general sobre como es la variabilidad espacio - temporal de la precipitación, tomando como ejemplo algunos estudios de los ciclos temporales de la precipitación con observaciones de mesoescala y de escala sinóptica en Colombia. Por lo reciente del uso de los radares meteorológicos en Colombia, no se ilustra la variabilidad espacio - temporal del campo de precipitación en las escalas de alta - resolución, ya que son pocos los ejemplos que pueden emplearse para ilustrar esta idea. No obstante, entre los ejemplos que se mencionan en esta sección, la caracterización del ciclo diurno incluye información pluviográfica de alta resolución temporal, la cual permite dar ejemplo de la variabilidad en las escalas más finas. En la Sección 4 se discuten dos desarrollos matemáticos que se han empleado ampliamente para la descripción espacio - temporal de la precipitación, que son representativos de los diferentes enfoques. Adicionalmente se presenta una breve introducción a los modelos de base física, que por su integralidad de conceptos, se establecen como precursores de los futuros modelos de precipitación. Se destaca que el primer desarrollo tratado en este artículo se asienta en las bases de los procesos estocásticos y el segundo en la teoría fractal. Sobre cada uno de los dos desarrollos se presentan las siguientes consideraciones: i) cuales han sido las dificultades para representar los patrones de precipitación en sus diferentes escalas de agregación a partir del conocimiento de las propiedades estadísticas de alguna de estas escalas, ii) la complejidad de describir la intermitencia de los patrones de precipitación y las relaciones entre escalas, y iii) señalar la falta de una base física en estas aproximaciones, que ayude a integrar el conocimiento de las propiedades físicas (termodinámicas) de la atmósfera con las propiedades estadísticas y geométricas del campo de precipitación. La Sección 5 se reserva para las conclusiones de este trabajo y para establecer algunos posibles direccionamientos futuros de investigaciones en torno al estudio de la precipitación. 


\section{Las ciencias hidrológicas y la precipitación}

Con el ánimo de aunar esfuerzos científicos que den respuestas a algunos de los interrogantes que se derivan del estudio de la precipitación, el Consejo Nacional de Investigación Americano (National Research Council, 1991) formuló dos retos científicos importantes respecto a la precipitación que aún están vigentes: i) ¿cómo integrar el comportamiento dinámico de los procesos hidrológicos en varias escalas espacio - temporales dada la presencia de una gran heterogeneidad natural? y ii) ¿cómo se puede establecer el enlace necesario y fundamental, entre los modelos determinísticos y estocásticos que describen la precipitación? Estas preguntas reflejan las preocupaciones de las ciencias hidrológicas frente a los problemas para describir (y predecir) la alta variabilidad espacial y temporal de la precipitación en las diferentes escalas en que ella es representada. Lo anterior identifica una necesidad vital en las ciencias hidrológicas: encontrar una teoría conceptual que integre los aspectos estadísticos, geométricos y físicos que caracterizan a la precipitación.

Los resultados obtenidos en múltiples publicaciones hidrológicas ponen en evidencia las limitaciones en la modelación de los procesos físicos no-lineales, que no corresponden a las idealizaciones teóricas que se apoyan en criterios de homogeneidad espacio - temporal y aún también, en la estacionariedad estadística de los procesos físicos. Esto ha conducido al abuso de la extrapolación de las mediciones a regiones que no cuentan con observaciones y consecuentemente, al desarrollo de diseños ineficaces o inadecuados de ingeniería. De alcanzarse una teoría física - matemática que represente simple y adecuadamente los procesos de precipitación en las múltiples escalas en que ellos son descritos, se optimizarían las parametrizaciones de los modelos meteorológicos e hidrológicos, se obtendrían mejores predicciones de corto y largo plazo con los modelos de cantidad y calidad del agua, se re-normalizarían los procedimientos de diseño en ingeniería y con ellos se alcanzaría un ahorro económico y de vidas en los proyectos de infraestructura y el desarrollo sostenible de las ciudades (National Research Council, 1991).

\section{La precipitación, su dinámica y variabilidad}

Una de las características más destacables de la precipitación es su alta variabilidad espacio - temporal, lo que dificulta la generalización de comportamientos típicos, aún sobre regiones no muy extensas. En la Figura 1 se ilustra el caso para Colombia donde la heterogeneidad de comportamientos de la precipitación media anual es bastante notable. Poveda, et al. (2002b) explican que la alta variabilidad de la precipitación en los Andes tropicales de Colombia se asocia a factores como: la orografía, las circulaciones locales, la humedad relativa, los procesos termodinámicos que inducen la alta inestabilidad atmosférica, la evaporación local, la interacción suelo - atmósfera y los cambios sobre los complejos convectivos de mesoescala. Las relaciones no-lineales de los anteriores descriptores determinan el alto grado de complejidad para explicar los procesos de precipitación y aún cuando se han realizado múltiples investigaciones, solamente se han llegado a caracterizaciones locales. No obstante, se debe poner en consideración que ante la incertidumbre actual en la medición y el procesamiento de los productos satelitales y de las estaciones de medición en tierra, subsiste un grado significativo de error en los estimativos hidrometeorológicos que pueden ser derivados de estos productos y en consecuencia, no se puede describir con precisión el estado dinámico de la precipitación del planeta y los procesos hidrológicos subyacentes (Adler, et al., 2011).

Anteriormente se mencionó que uno de los factores que explican la variabilidad de la precipitación es la orografía, pero la precipitación en si misma, es un factor importante que determina las características morfológicas del relieve y de las redes de drenaje. La erosión como factor principal

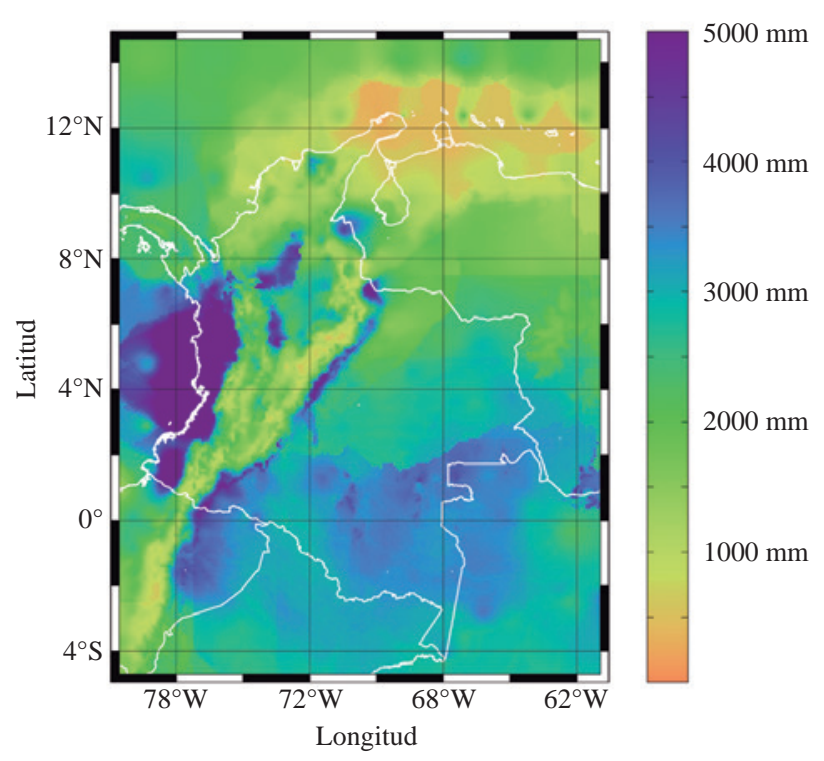

Figura 1. Campo de precipitación medio anual (en mm/año) para Colombia durante el periodo 1975 - 2006, el cual fue calculado por Hurtado (2009) a partir de 2.270 estaciones pluviográficas y las bases de datos del NCEP, GPCP, TRMM, GOES. La interpolación usa una modificación del modelo PRISM (Daly, et al., 1994, 2008) descrita en Hurtado (2009) y Hurtado \& Mesa (2014). La variabilidad de la precipitación sobre el territorio colombiano está determinado en gran medida por la orografía que conforman las cordilleras. La precipitación media anual más baja $(\approx 250 \mathrm{~mm} /$ año) se presenta al norte del país en la región del caribe y la precipitación media anual más alta $(\approx 12.200 \mathrm{~mm} / \mathrm{año})$ al occidente del mismo sobre la región pacífica. Sobre la región de la Orinoquía la precipitación media anual oscila entre los 1.800 y $3.000 \mathrm{~mm} /$ año, en la región amazónica entre los 3.000 y 4.000 mm/año y en el piedemonte llanero se alcanzan valores de hasta 7.000 mm/año. Sobre la región andina la heterogeneidad de las precipitaciones es bastante alta, las cuales varían entre los 800 y 3.000 mm/año. 
para la producción del paisaje es función de la intensidad y la concentración de la precipitación; por esto, la orografía es considerada como un predictor de la precipitación (Bookhagen \& Strecker, 2008). La investigación desarrollada por Bookhagen \& Strecker (2008) sobre la cordillera de los Andes en Suramérica, emplea una topografía digital de 90 metros de resolución y datos de intensidad de lluvia de la misión satelital TRMM (i.e. Tropical Rainfall Measuring Mission) para encontrar una clara relación entre la orografía y la precipitación que explica como los procesos erosivos están modulados por los picos de lluvia y como la precipitación está determinada por la barrera orográfica que define la cordillera de los Andes. Aún cuando existen indicios físicos que explican la relación entre orografía y precipitación, otros estudios como el desarrollado por Poveda, et al. (2005), concluyen que la variabilidad de la precipitación, asociada a la orografía, no está bien comprendida a todas las escalas espacio - temporales y es necesario obtener un mejor entendimiento de esta variabilidad para identificar las posibles amenazas sobre la biodiversidad que en ella se circunscribe.

Un primer escenario para comprender la variabilidad espacio - temporal de la precipitación, es mediante el análisis de los ciclos temporales de lluvia para diferentes escalas, por ejemplo las escalas inter-decadal, inter-anual, anual, intraanual y diurna. Por ejemplo, Poveda, et al. (2002b, 2005) caracterizan el ciclo diurno estacional de largo plazo de la precipitación en los Andes tropicales. Entre los resultados derivados del estudio del ciclo diurno, se resalta la alta variabilidad de los patrones obtenidos de la información de 51 estaciones ubicadas sobre los Andes tropicales colombianos. Se atribuye la variabilidad al papel que juega la topografía en el desarrollo de la precipitación, aunque no hay un patrón claramente identificado, ni una teoría que explique la existencia de regiones con un máximo nocturno de precipitación muy cerca de otras con lluvia predominantemente en las tardes. Sin embargo, Poveda, et al. (2002b, 2005) explican que los cambios estacionales encontrados en la precipitación máxima diurna, están asociados a la migración de la zona de convergencia intertropical (ZCIT).

La variabilidad de la precipitación de Colombia a escala anual se explica por la migración latitudinal de la ZCIT, la advección de humedad por los vientos alisios del Este y la dinámica del Chorro del Chocó y de los sistemas convectivos de mesoescala. En las Figura 2 y Figura 3 se ilustra el ciclo anual de los campos medios de precipitación sobre el territorio colombiano, los cuales fueron estimados por Hurtado (2009), haciendo uso de diferentes fuentes de información ${ }^{1}$. Desde un marco espacial, la Figura 2 permite identificar un periodo relativamente seco durante los meses

1 Hurtado (2009) empleó 2.270 estaciones pluviográficas y las bases de datos del NCEP, GPCP, TRMM, para la reconstrucción de los campos mensuales de precipitación para el periodo 1995 - 2006. de diciembre a marzo; un periodo de altas precipitaciones sobre el piedemonte andino y en las regiones de Amazona y Orinoquía durante los meses de abril a julio; un periodo de altas precipitaciones sobre la región del Pacífico y la cuenca media del río Magdalena durante los meses de agosto y noviembre. Por lo anterior, el ciclo anual de la precipitación en Colombia se caracteriza por contar con dos periodos de lluvias intensificadas que se explican por el paso de la zona de convergencia intertropical (ZCIT) sobre el país (Hurtado \& Mesa, 2014; Hurtado, 2009). Se puede apreciar en la Figura 2 que durante todo el año se presentan precipitaciones sobre la región del Pacífico, que obedecen fundamentalmente a lo que previamente se indicó como la advección de humedad por los vientos alisios del Este y la dinámica del Chorro del Chocó (Poveda \& Mesa, 1999).

Desde un marco temporal, la Figura 3 describe el ciclo anual para cuatro locaciones específicas dentro del territorio colombiano, tomando como información base, los campos de precipitación estimados por Hurtado (2009). En el recuadro superior, se presenta el ciclo anual de una de las regiones más secas de Colombia (i.e. Riohacha, Guajira) donde la precipitación media anual es de $587 \mathrm{~mm}$, en los recuadros intermedios, el ciclo anual de dos municipios localizados en la región andina (i.e. Medellín (Antioquia) y Bogotá) y en el recuadro inferior, se encuentra el ciclo anual de la región más húmeda del país (i.e. Quibdó, Chocó), cuya precipitación media anual es de $8.016 \mathrm{~mm}$. La Figura 3 nos señala la alta variabilidad de la cantidad total del agua precipitada entre las distintas locaciones, no obstante, existe una característica sobresaliente y es la simetría distinguible (bimodal) del ciclo anual de precipitación, para las cuatro locaciones allí indicadas. En el ciclo anual del municipio de Quibdó se distingue una amplificación sustancial de las precipitaciones respecto a las otras locaciones, dado que sobre esta región se ejerce la fenomenología climática conocida como el Chorro del Chocó (Poveda \& Mesa, 1999), la cual aumenta la advección de humedad en la región del Pacífico colombiano.

Para Colombia y regiones aledañas, la variabilidad interdecadal de la precipitación es gobernada por fenómenos macro-climáticos, como son: la Oscilación Decadal del Pacífico $^{2}$ - PDO, la Oscilación del Atlántico Norte ${ }^{3}$ NAO y la Oscilación Multidecadal del Atlántico Norte ${ }^{4}$

2 La Oscilación Decenal del Pacífico es un patrón de variabilidad climática en el Océano Pacífico que tiene un periodo de oscilación decadal de 20 30 años y que mantiene características similares a las dos fases del ENSO (Hurtado, 2009).

3 La Oscilación del Atlántico Norte es la alternación de la masa atmosférica entre las regiones subtropical y subpolar del Océano Atlántico que se caracteriza por variaciones en el gradiente de presiones al nivel medio del mar, en los vientos del oeste en las latitudes medias, en las temperaturas superficiales del océano y en el clima de las regiones continentales (Hurtado, 2009).

4 La Oscilación Multidecadal del Atlántico Norte es un patrón de variabilidad de la temperatura superficial centrado en el norte del Océano Atlántico que se ha asociado a la ocurrencia de sequías en Sahel (África), a la variabilidad de la precipitación del nordeste de Brasil y a la frecuencia de huracanes en el Océano Atlántico (Mesa, 2007). 

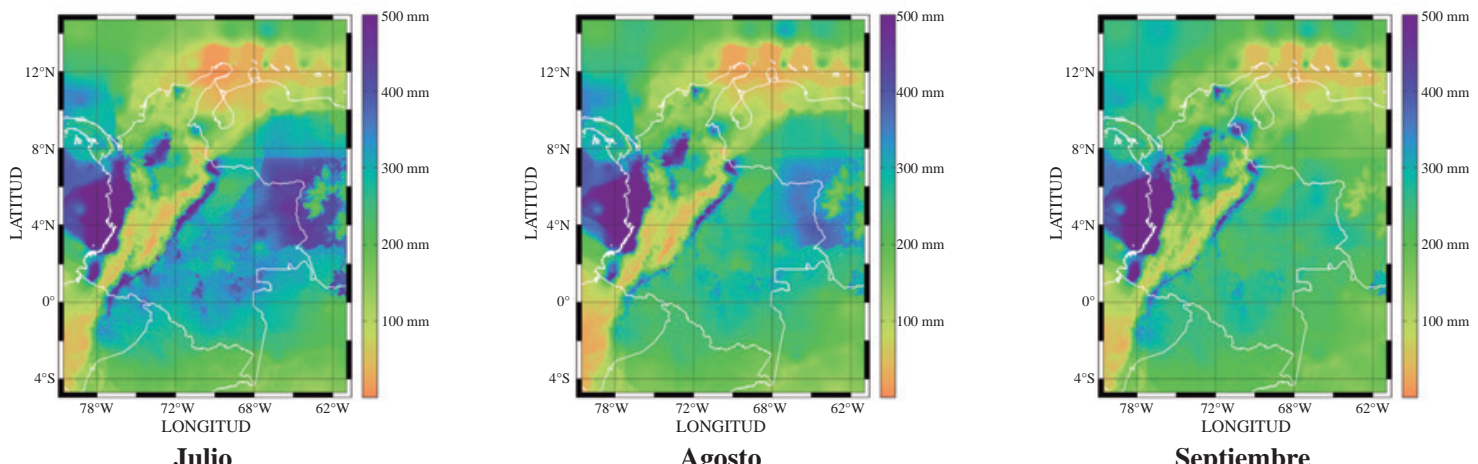

Septiembre
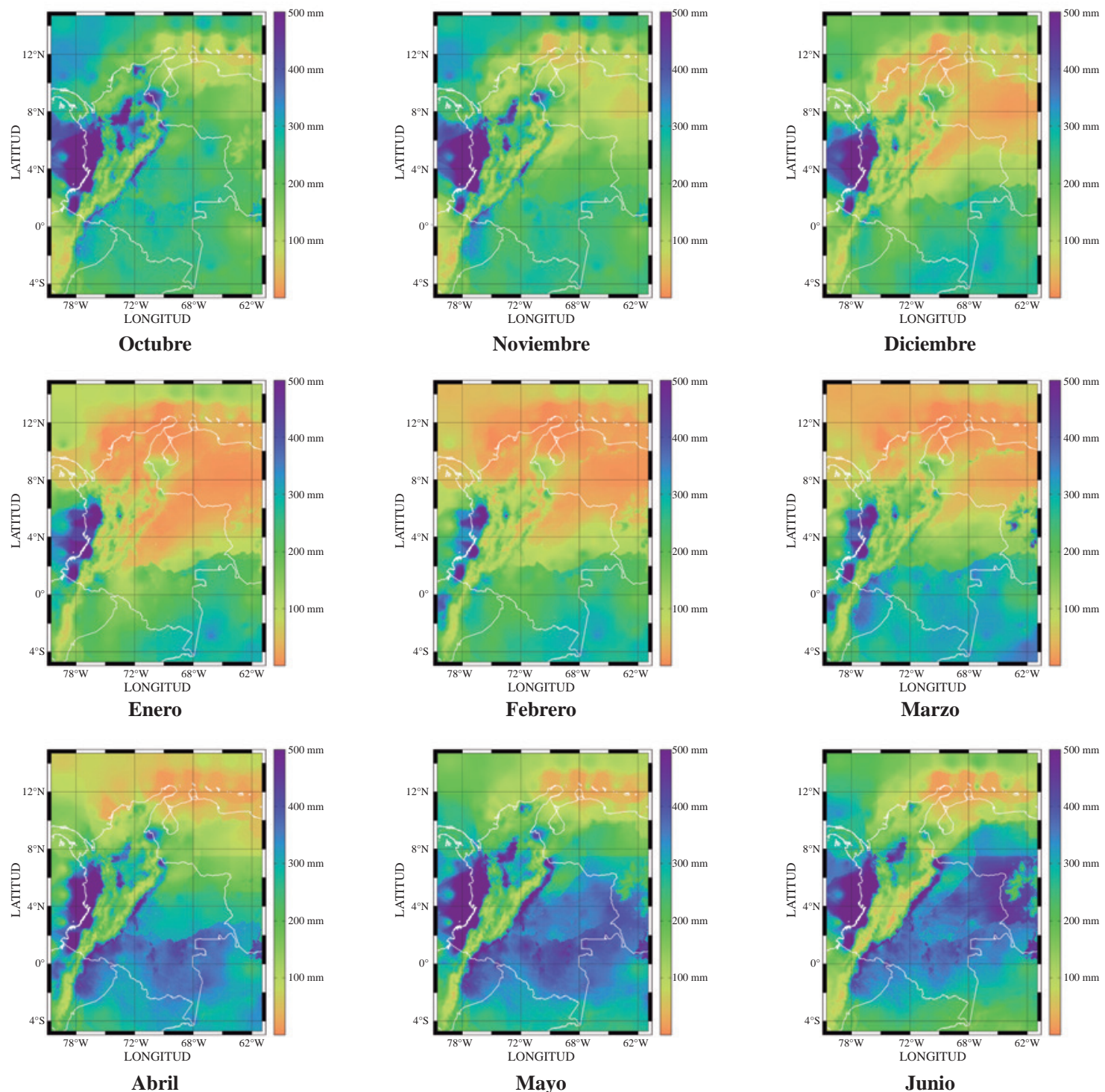

Figura 2. Campos de precipitación medios mensuales (en mm/mes) para Colombia durante el periodo 1975 - 2006, los cuales fueron calculados por Hurtado (2009) a partir de 2.270 estaciones pluviográficas y las bases de datos del NCEP, GPCP, TRMM, GOES. La interpolación usa una modificación del modelo PRISM (Daly, et al., 1994, 2008) descrita en Hurtado (2009) y Hurtado \& Mesa (2014).

AMO (Hurtado, 2009; Mesa, 2007; Poveda, 2004). Las observaciones de precipitación para Colombia al parecer no muestran una tendencia distintiva de la variabilidad decadal, sin embargo, otras variables climatológicas identifican cambios en la fase y la amplitud del ciclo anual y semianual, implicando un desplazamiento de las temporadas 

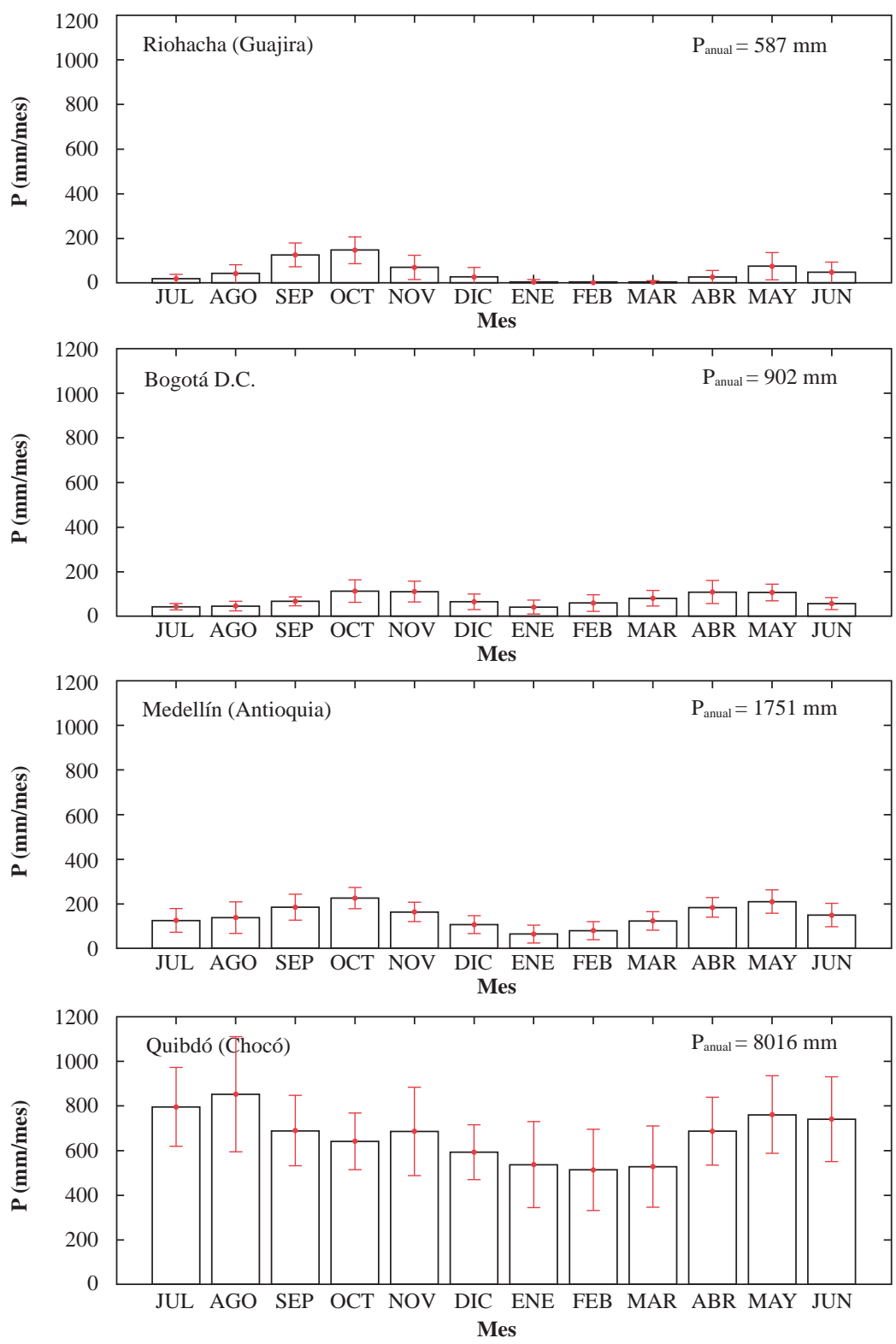

Figura 3. Ciclo anual de la precipitación media mensual (en mm/mes) para los municipios de Medellín (Antioquia), Bogotá, Riohacha (Guajira) y Quibdó (Chocó), durante el periodo 1975 - 2006, los cuales fueron estimados a partir de los campos de precipitación calculados por Hurtado (2009). Una característica distinguible del ciclo anual de la precipitación de Colombia es su estructura bimodal, la cual identifica la migración de la ZCIT sobre el territorio colombiano. Así mismo, la amplia variabilidad de la precipitación en Colombia es un reflejo de su complejidad espacial. Note la uniformidad de la escala de la gráfica.

de lluvia en el país (Poveda, 2004). En la escala inter-anual, la precipitación es controlada por la ocurrencia del evento ENSO (El Niño Oscilación del Sur5). Múltiples estudios han sido desarrollados en Colombia tomando como eje central

5 El ENSO (El Niño Oscilación del Sur) es un evento macroclimático recurrente, que se presenta aperiódicamente con un pico de frecuencia al rededor de los 4 años y se caracteriza por el calentamiento anormal de las aguas superficiales del Océano Pacífico tropical Este, anomalías en la presión atmosférica en las mismas zonas, aumento de las precipitaciones en algunas regiones del planeta, la profundidad de la termoclina aumenta en el Pacífico tropical Este y el nivel del mar sube (Hoyos \& Webster, 2006). el análisis de la influencia del ENSO en sus fases cálida (El Niño) y húmeda (La Niña) sobre el ciclo diurno y anual de la precipitación (Poveda, et al., 2002a, b, c; Mesa, et al., 1997). Los estudios han determinado, de manera general, que El Niño está asociado con anomalías negativas en la cantidad de lluvia y La Niña con anomalías positivas de la misma (Poveda, et al., 2002a; Poveda, 2004). Los resultados muestran que durante La Niña la precipitación total diaria puede aumentar hasta un $40 \%$ en comparación con los años considerados como normales y hasta un $48 \%$ más en relación a los eventos El Niño (Poveda, et al., 2002a, 2005). 
Como respaldo a lo anteriormente indicado, la Figura 4 presenta los campos medios mensuales de precipitación durante el evento El Niño de los años 1997 - 1998. Aquí se distingue una disminución sustancial en la cantidad total de precipitación en relación al campo medio anual que se ilustró en la Figura 2. Durante los meses de diciembre a marzo, Colombia recibió el mayor impacto del evento El Niño, ocasionando conflictos en el uso consuntivo del
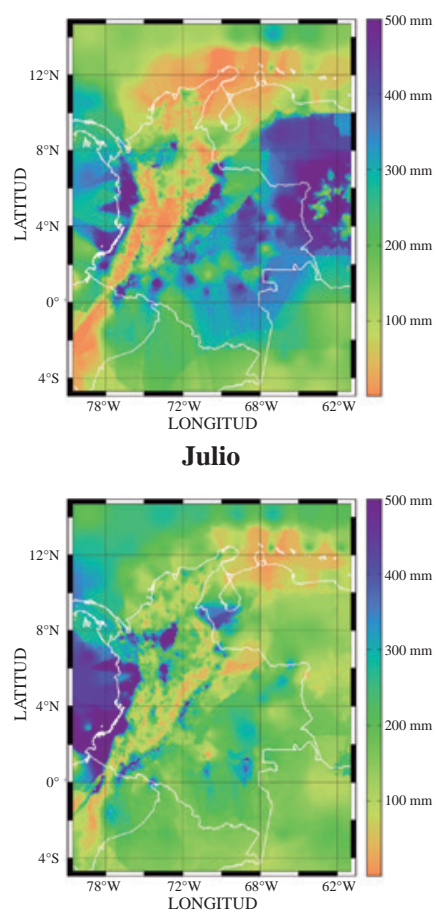

Octubre

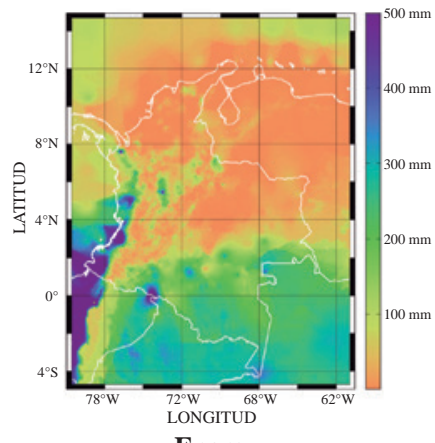

Enero

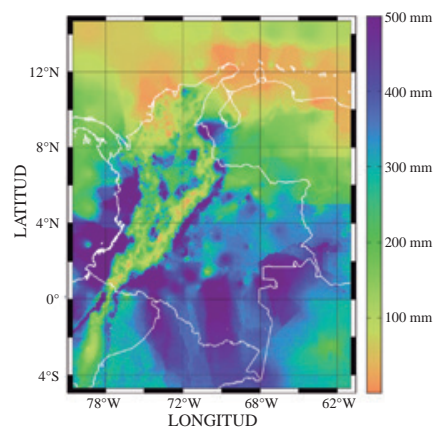

Abril
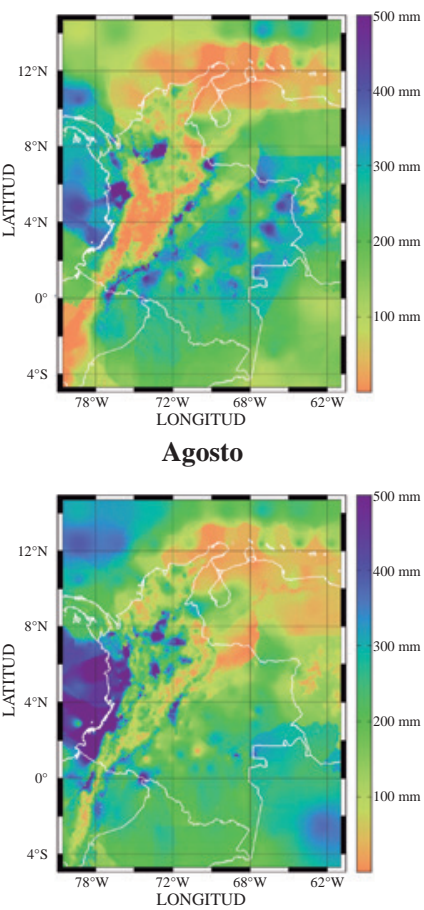

Noviembre

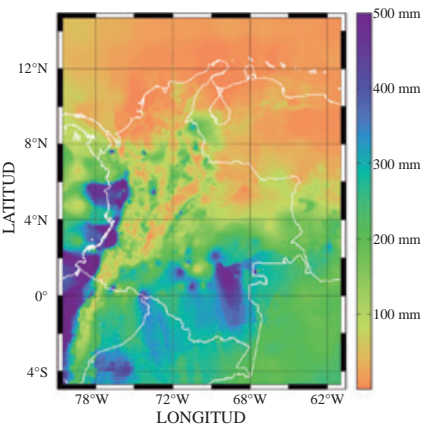

Febrero

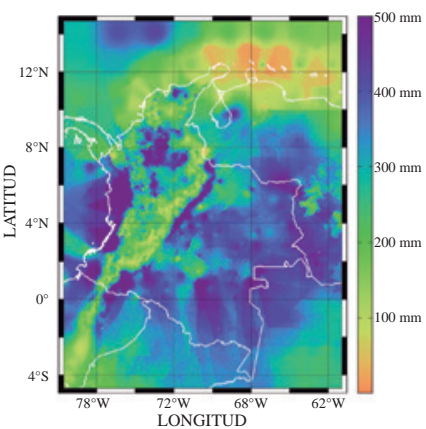

Mayo

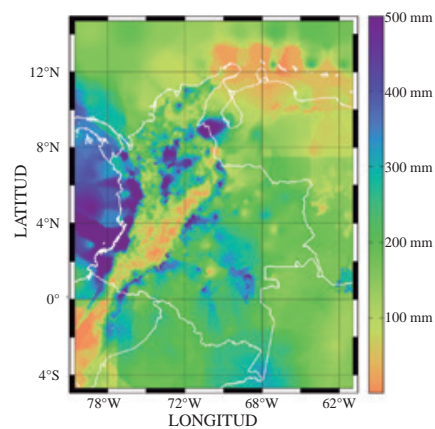

Septiembre

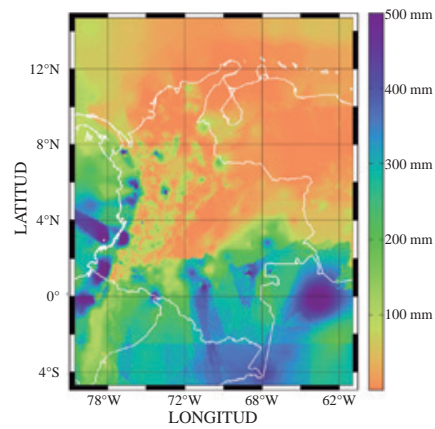

Diciembre

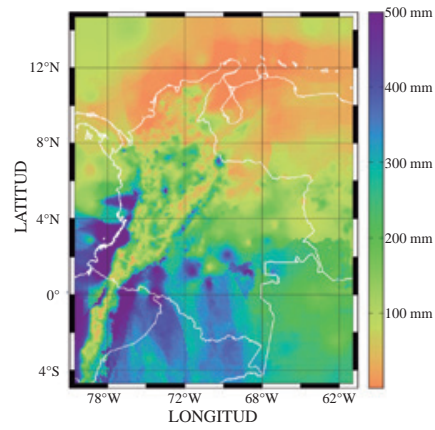

Marzo

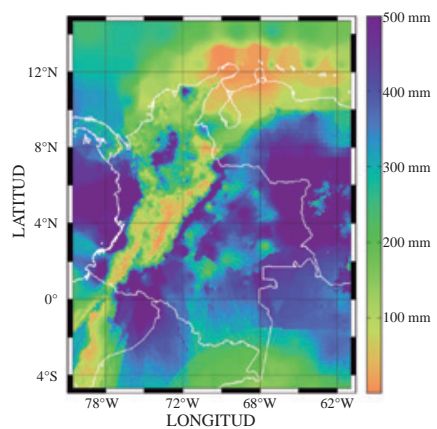

Junio

Figura 4. Campos mensuales de precipitación medios mensuales (en mm/mes) para Colombia durante el evento El Niño de 1997-1998, los cuales fueron calculados por Hurtado (2009) a partir de 2.270 estaciones pluviográficas y las bases de datos del NCEP, GPCP, TRMM, GOES. La interpolación usa una modificación del modelo PRISM (Daly, et al., 1994, 2008) descrita en Hurtado (2009) y Hurtado \& Mesa (2014). 
agua, la disminución de niveles en los embalses, pérdidas en las cosechas de productos agrícolas, entre otros más. En la Figura 1S, http://www.raccefyn.co/index.php/raccefyn/ article/downloadSuppFile/196/996, se presenta la contracara del evento anterior, conocido como La Niña. En esta figura, se ilustra el evento de los años 1999 y 2000, el cual se caracterizó por ser uno de los eventos de mayor duración y que consistió de dos momentos temporales donde se intensificaron las precipitaciones. Es importante resaltar que durante un evento La Niña, los conflictos por el agua adoptan otro carácter al que se presenta durante un evento El Niño, puesto que el exceso de agua contribuye al desarrollo de inundaciones, a la susceptibilidad de falla de taludes, a la marchites de las plantas por exceso en la capacidad de campo del suelo, etc.

A la escala intra-anual, la variabilidad es modulada por la Oscilación Maden - Julian ${ }^{6}$ (MJO), las ondas tropicales del Este, los huracanes sobre el Caribe y el Pacífico oriental y los frentes fríos procedentes desde ambos hemisferios en época de invierno (Poveda, 2004). Para Colombia se destaca que el ciclo diurno de la lluvia es más activo durante la fase Oeste ${ }^{7}$ de la MJO y se presenta un incremento de la precipitación total diaria de hasta un $77 \%$ en relación al valor medio de la misma (Poveda, et al., 2002a).

Los estudios sobre la MJO reconocen que es necesario determinar el origen de las oscilaciones y explicar los mecanismos físicos de interacción, para tener un claro panorama de las implicaciones en el estado del clima y de la precipitación sobre el territorio colombiano (Poveda, et al., 2002a; Hoyos \& Webster, 2006). Todas las observaciones que aquí se han destacado de los estudios sobre la precipitación en Colombia en sus diferentes escalas temporales, nos advierten del alto grado de complejidad para explicar su variabilidad y dinámica; por lo tanto, aún se mantienen abiertos los interrogantes sobre que herramientas y procedimientos matemáticos convendrían ser utilizados para acercarnos a un mayor entendimiento de este proceso físico.

\section{Modelos descriptivos de la precipitación}

\subsection{Los modelos estocásticos}

Como ha podido evidenciarse en las Figuras 2 a 4, la precipitación se caracteriza por la alta variabilidad de sus observaciones tanto en el espacio como en el tiempo. Si concentramos nuestra atención en la variabilidad temporal, es decir, en el patrón que se observa en las series de tiempo que son registradas en las estaciones pluviográficas, como se

6 La Oscilación Maden - Julian es un fenómeno climático que se caracteriza por la presencia de oscilaciones en diferentes variables climáticas a la escala temporal entre 25 y 80 días, la cual se reconoce por la presencia de anomalías en la precipitación (Hoyos \& Webster, 2006).

7 Se considera la fase Oeste (o positiva) de la MJO, las épocas en las que la velocidad del viento zonal es mayor a una desviación estándar en relación a una serie índice (Poveda, et al., 2002a). ilustra en la Figura 2S, http://www.raccefyn.co/index.php/ raccefyn/article/downloadSuppFile/196/997. Se puede llegar a la conclusión que expresa Rodríguez-Iturbe (1991), al decir que la precipitación es un proceso físico que proviene de un sistema determinístico altamente no-lineal, cuyos patrones conservan una apariencia similar a la generada por un proceso aleatorio. La idea de enmarcar la precipitación como una variable física aleatoria, incentivo a que los primeros intentos por describir la precipitación consistieran en buscar un tipo de proceso aleatorio que se adapte a todos los conjuntos de datos observados, pero ante la marcada variabilidad de las observaciones tanto en el espacio como en sus diferentes escalas de agregación, no se ha logrado determinar la existencia de un único proceso aleatorio, y lo que es más grave, no hay consistencia cuando se cambia la escala de agregación. Con base en lo anterior, la consideración que describe la precipitación como un proceso físico que puede ser explicado por funciones aleatorias no ha producido una teoría satisfactoria. Aunque el concepto general es adecuado para caracterizar la imposibilidad de hacer predicciones, es incompleta, no explica la heterogeneidad espacio - temporal de la precipitación en todas sus escalas y requiere hipótesis especificas al lugar de estudio y a la escala espacial y temporal usada en el modelo.

Rodríguez-Iturbe, Gupta \& Waymire (1984) señalan que la mayor dificultad de los modelos estocásticos está en la estimación de sus parámetros. Uno de los primeros modelos estocásticos que fue diseñado para la representación de eventos de tormenta es el denominado modelo de ruido blanco de Poisson (Rodríguez-Iturbe, et al., 1984). Aquí se representa el número de tormentas de lluvia $N(t)$ como una secuencia de pulsos instantáneos que son gobernados por un proceso de Poisson ${ }^{8}$ con parámetro $\lambda$. El primer problema que se puede identificar de este proceso está relacionado con la estimación de la covarianza ${ }^{9}$ evaluada sobre la secuencia de valores discretos de lluvia acumulada $\left(Y_{i}\right)$. La covarianza observada es significativamente mayor a la correspondiente en el modelo, en donde por definición los incrementos $\left(Y_{i}\right)$ son independientes, luego $\operatorname{Cov}\left[Y_{i}, Y_{i}\right]=0$. Como se puede observar en la Figura 5, la función de autocorrelación ${ }^{10}$, aunque decae rápidamente para los registros de precipitación agregados en las menores escalas de agregación temporal, no es cero para todos los rezagos. Debe tenerse en cuenta

8 En un proceso de Poisson $N(t)$ representa el número de pulsos (eventos) en el intervalo $(0, t)$. Hay varias definiciones, una de las más simples establece que (i) los incrementos son independientes, es decir que $N\left(t_{2}\right)-N\left(t_{1}\right)$ es independiente estadísticamente de $N\left(t_{4}\right)-N\left(t_{3}\right)$ para $t_{1}<t_{2}<t_{3}<t_{4}$; y (ii) las probabilidades siguen la distribución $\operatorname{Pr}\{N(t)=n\}=p_{n}=(1 / n !)(\lambda t)^{n} e^{-\lambda t}$ con parámetro $\lambda$ (Feller, 1967; p.292, 446; Bhattacharya \& Waymire, 2009; p.261).

9 La covarianza de dos variables aleatorias $X$ e $Y$ se define como $\operatorname{Cov}[X, Y]=E\left[\left(X-\mu_{x}\right)\left(Y-\mu_{y}\right)\right]$, donde $E$ representa la esperanza matemática y $\mu_{X}=E[X]$ es el valor esperado de la variable aleatoria (Feller, 1967; p.230).

10 Puede asociarse la función de correlación con la covarianza del proceso mediante la expresión $\rho(\mathrm{X}, \mathrm{Y})=\operatorname{Cov}[X, Y] /\left(\sigma_{x} \sigma_{y}\right)$ (Feller, 1967). 

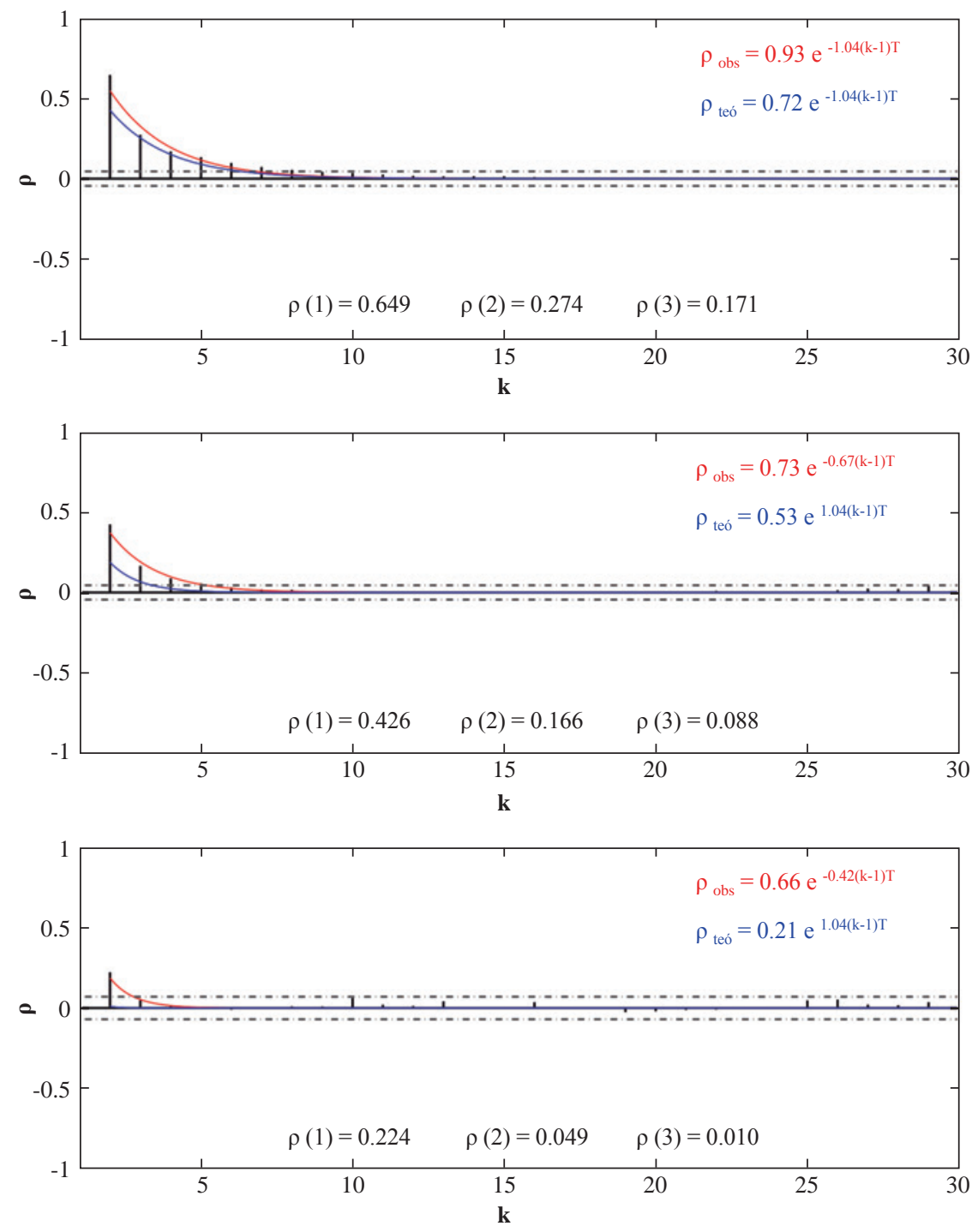

Figura 5. Función de correlación de valores totales de precipitación (en mm) del año de 1999 para las escalas de agregación temporal: (a) $\mathrm{T}=30 \mathrm{~min}$, (b) $\mathrm{T}=60 \mathrm{~min}$ y (c) $\mathrm{T}=3 \mathrm{~h}$, los cuales fueron medidos en la estación pluviográfica Bosa Barreno (Bogotá) y las curvas de ajuste de la función de correlación de las observaciones (línea de color rojo) y la expresión teórica indicada en Rodríguez-Iturbe, et al. (1984, Ec. 29) (línea de color azul). Las líneas punteadas representan los intervalos de confianza para el cual los valores de la función de correlación son significativamente diferentes de cero; estos intervalos de confianza fueron estimados para un nivel de significancia del 95\%, así su magnitud es calculada como 1.96 veces el error estándar para grande rezagos de la función de correlación (Hipel \& McLeod,1994, p.73). En esta figura $k>2$ representa el rezago (o desfase) para el cual es calculada la función de correlación. El decaimiento exponencial de la función de correlación de las intensidades de precipitación es un aspecto considerado en el modelo de pulsos rectangulares de Poisson (Rodríguez-Iturbe, et al., 1984; Rodríguez-Iturbe, et al., 1987), sin embargo, el exponente de decaimiento que se muestra en cada una de las gráficas, no se mantiene constante para todas escalas. Por lo cual, las limitaciones de los modelos de Poisson para la lluvia, residen en que los parámetros obtenidos para una escala de agregación pueden no llegar a ser funcionales para describir procesos en otras escalas.

que las funciones de correlación que se muestran en la Figura 5 no representan la correlación entre el número de aguaceros $\rho\left(N_{i}, N_{i+k}\right)$ en diferentes escalas temporales $T$, sino la correlación entre el total de precipitación $\rho\left(Y_{i}, Y_{i+k}\right)$.

Ahora, la comparación vamos hacerla con un modelo de Poisson asumiendo que el total de precipitación $\left(Y_{i}\right)$ que ocurre en cada evento, es una variable aleatoria con una determinada distribución, independiente para cada evento, lo que en la literatura se conoce como un proceso de Poisson marcado; o la variante llamada pulsos rectangulares de Poisson (Rodríguez-Iturbe, et al., 1987, Rodríguez-Iturbe, 1986), en donde la duración y la intensidad (constante durante la duración) del evento son variables aleatorias independientes. En ambos casos la conclusión de la comparación es la misma, porque la estructura de dependencia es 
heredada del proceso de Poisson, y sólo en el modelo de pulsos rectangulares de Poisson (cuando las distribuciones para la duración y la intensidad son exponenciales) hay lugar a un decaimiento exponencial de la correlación ${ }^{11}$, como se observa en la Figura 5, pero la tasa de decaimiento exponencial $(\eta)$ deberá ser la misma, independiente del nivel de agregación temporal (i.e. en el ejemplo este valor es igual a 1.04). Rodríguez-Iturbe (1986) y Rodríguez-Iturbe, et al. (1987) señalan la conclusión que hemos resaltado en el sentido que los parámetros estimados para un determinado nivel de agregación producen resultados deficientes si se aplican a otras escalas temporales. La Figura 2S ilustra la relación entre la intensidad máxima y la escala de agregación para las series de tiempo observadas en Bosa Barreno en el año 1999 y las simuladas con el modelo de pulsos rectangulares de Poisson desde la escala de 30 minutos. Estos resultados reiteran la imposibilidad del modelo para capturar completamente la estructura estadística y geométrica de las observaciones, considerando que en este caso particular, el modelo preserva el decaimiento exponencial de la relación, pero sobreestima las intensidades máximas de precipitación.

Hay modelos estocásticos más generales, como son aquellos que proceden de la familia de modelos de Neyman - Scott. En el modelo denominado ruido blanco de Neyman - Scott (Rodríguez-Iturbe, et al., 1984; Rodríguez-Iturbe, 1986), se consideran que los tiempos de arribo de las tormentas son gobernados por un proceso de Poisson con parámetro $\lambda$ y las tormentas tienen un número aleatorio de pulsos asociados que es representado por otro proceso aleatorio puntual. Por otra parte, para obtener los cuatro parámetros que constituyen al modelo de Neyman - Scott, es necesario implementar un procedimiento iterativo para resolver simultáneamente el sistema de ecuaciones que involucra a los parámetros y comparar las respuestas de las varianzas teórica y estimada.

Puede verse, que en la medida en que el proceso estocástico se hace más general en su capacidad para describir adecuadamente los procesos de precipitación observados, el número de parámetros aumenta y del mismo modo, la complejidad para su estimación; por otra parte, estos modelos resultan ser mas eficientes para la descripción de patrones en las escalas menores a la horaria y aún se mantienen las dificultades con los estimativos que resultan de las transformaciones de escala ${ }^{12}$. Es de resaltar que en

11 En este caso la función de correlación adquiere la forma $\rho\left(Y_{i}, Y_{k}\right)=C e$ $-\eta(k-1) T$, siendo $\eta$ un parámetro asociado a la duración media de los eventos de lluvia y que representa la tasa de decaimiento, $T$ la escala temporal de los datos y $C$ una constante.

12 Otros modelos estocásticos como es el modelo STREAP (Realizaciones Espacio - Temporales de Precipitación Areal), el cual fue introducido por Paschalis, et al., (2013), es capaz de reproducir algunas características esenciales del campo de precipitación mediante la descripción del arribo de las tormentas, la evolución temporal de precipitación areal dentro de la tormenta y la evolución temporal de la estructura bidimensional de la tormenta. Pese a que este modelo mejora en la capacidad para reproducir las propiedades estadísticas del campo de precipitación, siguen existiendo adición a sus dificultades conceptuales, la implementación de estos modelos estocásticos para la representación de patrones temporales de precipitación resultaran ineficaces para Colombia por razones prácticas, puesto que la mayor parte de las mediciones que se efectúan en las estaciones se dan a una resolución temporal de 24 horas, limitando así, la confiabilidad de los resultados que puedan derivarse de estos modelos.

\subsection{Los modelos fractales}

Rodríguez-Iturbe (1991) afirma que los patrones temporales de precipitación aunque irregulares, preservan una cierta estructura, cuya clave está presente en la relación entre las irregularidades de las escalas. Esta relación puede ser comprendida mediante la propiedad fractal de autosemejanza, que caracteriza a los objetos por la invariancia respecto a la traslación y cambio de escala (Feder, 1988). Una manera de caracterizar el grado de irregularidad de las fluctuaciones en los patrones de precipitación es mediante el uso de objetos geométricos denominados: medidas multifractales. Estos objetos geométricos se conforman por subconjuntos de objetos fractales, que se caracterizan por tener un espectro de exponentes de escalamiento (Feder, 1988). Una manera de evidenciar esta característica geométrica en los registros de precipitación se puede observar en el ejemplo que se ilustra en el recuadro superior de la Figura 6. Aquí, Los momentos de orden $k$ de la precipitación total a la escala $T$, denotados por $m_{k}(T)=E\left[X^{k}(T)\right]$, muestran una relación potencial con la escala, es decir $m_{k}(T) \propto \mathrm{T}^{\theta k}$. De esta relación resulta un exponente de escalamiento $\theta_{k}$ que depende del orden del momento $k$. Si la serie de tiempo de precipitación que se describe con la relación $m_{k}(T) \propto \mathrm{T}^{\theta k}$, correspondiera a la de un objeto monofractal, i.e. un objeto fractal con escalamiento simple, la dependencia de $\theta_{k}$ en $k$ sería lineal, como lo indica la línea punteada del recuadro inferior de la Figura $6^{13}$. Sin embargo, se puede apreciar que para la serie de tiempo de precipitación que se toma como ejemplo, se presenta un crecimiento sublineal en la relación entre $k$ y $\theta_{k}$, que intuitivamente podría indicarnos que se está tratando no solo con un objeto multifractal, sino también con un proceso físico no-lineal. Es importante destacar, que el grado de multifractalidad en la serie de tiempo puede determinarse, parcialmente, si observamos una tendencia del exponente $\theta_{k}$ hacia valores cercanos a cero. Otras herramientas como son el espectro multifractal y la función de codimensión, permiten caracterizar de mejor forma la estructura multifractal de un conjunto de datos. En el contexto del estudio de la estructura multifractal de la precipitación se recomienda revisar los trabajos efectuados por Lovejoy \& Schertzer (1995), Gómez \& Poveda (2008) y

limitaciones con la tractabilidad matemática del modelo, la poca o no preservación de las propiedades geométricas del campo de precipitación y la ausencia de una base física en sus parámetros.

13 En el recuadro inferior de la Figura 6, se ilustra una función conocida bajo el nombre de función de estructura. 

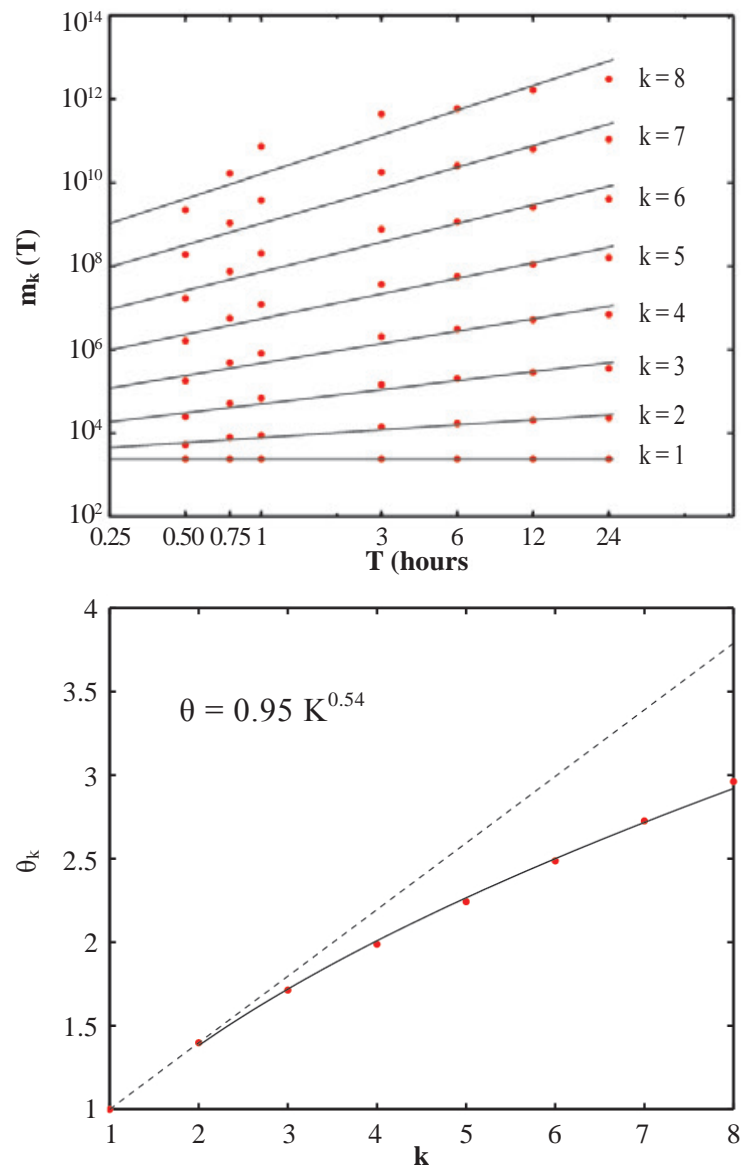

Figura 6. Para la serie de tiempo de valores de precipitación (en mm) medidos en la estación pluviográfica Bosa Barreno (Bogotá) durante el periodo 1995-1999 (ver Figura 3S, http://www.raccefyn.co/index. php/raccefyn/article/downloadSuppFile/196/998), el cuadro superior muestra los primeros ocho momentos estadísticos $m_{k}(T), k=1,2$, ..., 8, en función de la escala de agregación temporal T = 30 min, 45 min, 1h, 3h, 6h, 24h y el cuadro inferior, la función de estructura correspondiente, $\theta_{k}$ vs. $k$. En el marco conceptual de multifractales universales (Lovejoy \& Schertzer, 1995; Schertzer \& Lovejoy, 2011), un campo es multifractal si un arbitrario momento $m_{k}$ escala de la forma: $m_{k}(T) \approx T^{\theta k}$, donde $\theta_{k}$ representa una función de escalamiento de los momentos estadísticos, que caracteriza la variabilidad del campo para diferentes escalas de agregación temporal. El comportamiento bilineal que describe la función de estructura para las observaciones de precipitación de la estación Bosa Barreno, permite evidenciar la existencia de una estructura multifractal en la información para el rango de escalas que aquí se indican.

Peñaranda (2008), para mayores detalles sobre los métodos de construcción del espectro multifractal y la caracterización de campos de precipitación desde este enfoque.

Dada la existencia de una estructura multifractal en los patrones de precipitación, la comunidad científica no tardó en introducir modelos que preservarán tanto las propiedades estadísticas de las observaciones, como también las propiedades multifractales encontradas en ellas. Uno de los primeros modelos conceptuales que empleó el concepto de multifractalidad es el denominado modelo de cascadas aleatorias, introducido por Gupta \& Waymire (1993) y posteriormente implementado por Over \& Gupta (1994, 1996) y Over (1995). Este modelo a diferencia de los modelos estocásticos, requiere de muy pocos parámetros para representar la intermitencia en el dominio espacio - temporal, sin embargo, las bases conceptuales del modelo se apoyan en la teoría de funciones aleatorias para la construcción del campo y en virtud a ello, existen dificultades en la validación de las hipótesis que giran en torno al tipo de generador aleatorio utilizado en el proceso de construcción de las cascadas multiplicativas.

Otro de los modelos que usa la teoría multifractal pero con naturaleza enteramente determinística, fue introducido por Puente (1996); Puente \& Obregón (1996), el cual se denomina Aproximación Fractal - Multifractal (FMF). En este modelo los patrones observados son estimados a partir de la construcción de una función de interpolación fractal ${ }^{14}$. Es claro que la geometría Euclidiana y las funciones matemáticas elementales son la base de los métodos tradicionales para el manejo e interpretación de datos (Barnsley, 2006), como por ejemplo el método de mínimos cuadrados. Si en lugar de emplear estos métodos para interpolar un grupo de puntos, se utilizasen combinaciones lineales de funciones elementales de la forma:

$$
W_{n}\left(\begin{array}{l}
x \\
y
\end{array}\right)=\left(\begin{array}{ll}
a_{n} & 0 \\
c_{n} & d_{n}
\end{array}\right)\left(\begin{array}{l}
x \\
y
\end{array}\right)+\left(\begin{array}{l}
e_{n} \\
f_{n}
\end{array}\right)
$$

se obtiene lo que es denominado como: función de interpolación fractal (Barnsley, 2006), en donde $a_{n}, c_{n}, d_{n}$, $e_{n}$ y $f_{n}$ son los parámetros fundamentales de esta función. Si consideramos un conjunto de tres pares de puntos $\{(0,0)$, $(1 / 2,1),(1,0)\}$, como el que se ilustra en el recuadro superior izquierdo de la Figura 7 (puntos de color rojo), los cuales son interpolados a través de dos sistemas de ecuaciones lineales (o mapas afines) $W_{1} \mathrm{y} W_{2}$, cuyos parámetros son indicados a la derecha de la misma figura, se consigue una interpolación lineal entre los pares de puntos. Para esta interpolación el parámetro $\left|d_{n}\right|$ se fijó en un valor $z=0.0$. Ahora, si el parámetro $\left|d_{n}\right|$ se fija en un valor $z=0.8$ (ver recuadro inferior izquierdo de la Figura 7); se consigue una interpolación no-lineal, más compleja e intrincada. Para este último caso, la dimensión geométrica que describe la trayectoria entre los puntos no es una línea recta con dimensión igual a 1.0, sino en vez, un complejo sistema de objetos lineales inter-relacionados que tienen una dimensión no entera igual a 1.7 (en este caso); razón por la cual esta función de interpolación adquiere el nombre de fractal ${ }^{15}$.

14 Ver la referencia: Barnsley (2006) para mayor detalle del proceso de construcción de las funciones de interpolación fractal.

15 El término fractal se le atribuye a Benoit Mandelbrot, quien lo empleó para describir objetos geométricos irregulares o fragmentados. Una introducción a la geometría fractal puede consultarse en la referencia: Mandelbrot (1982). 


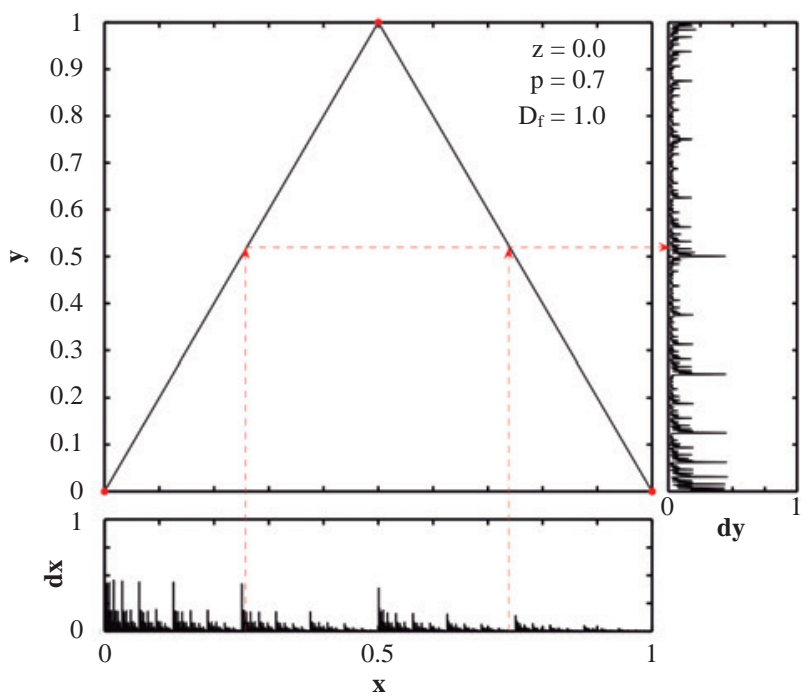

\begin{tabular}{ccc}
\hline & \multicolumn{2}{c}{ Parámetros } \\
\hline & $w_{1}$ & $w_{2}$ \\
a & 0.50 & 0.50 \\
c & 1.00 & -1.00 \\
d & 0.00 & 0.00 \\
e & 0.00 & 0.50 \\
f & 0.00 & 1.00 \\
p & 0.70 & 0.30 \\
\hline
\end{tabular}

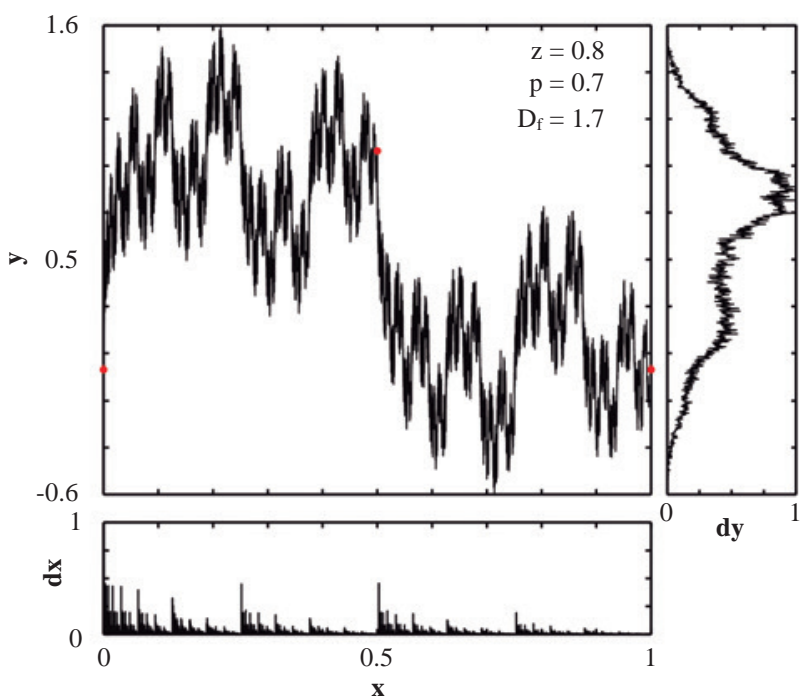

\begin{tabular}{ccc}
\hline & \multicolumn{2}{c}{ Parámetros } \\
\hline & $w_{1}$ & $w_{2}$ \\
a & 0.50 & 0.50 \\
c & 1.00 & -1.00 \\
d & 0.80 & -0.80 \\
e & 0.00 & 0.50 \\
f & 0.00 & 1.00 \\
p & 0.70 & 0.30 \\
\hline
\end{tabular}

Figura 7. La aproximación FMF se describe a través de tres elementos constitutivos que pueden ser observados en las imágenes: (a) la medida multifractal, (b) la función de interpolación fractal y (c) la medida derivada. La línea roja discontinua nos orienta sobre el sentido en que deben leerse las gráficas. La función de interpolación de las gráficas aquí indicadas, se construyeron para los puntos: $\{(0,0),(0.5,1)$, $(1,0)\}$, fijando un valor del parámetro $\left|d_{n}\right|=z$. En la aproximación FMF, los patrones geofísicos pueden derivarse desde la proyección de una medida multifractal sobre una función de interpolación fractal. Este concepto puede también apreciarse en las gráficas de la Figura 4S, http://www.raccefyn.co/index.php/raccefyn/article/downloadSuppFile/196/1001, del material suplementario.

Durante la construcción de la función de interpolación fractal, dos medidas de probabilidad son generadas mediante el conteo de frecuencias de los puntos definidos en las coordenadas $x$ e $y$ de la trayectoria de la función de interpolación (Puente, 1996). Para las funciones de interpolación que se ilustran en la Figura 7, en $x$ se obtiene una medida $(d x)$ binomial ${ }^{16}$ multifractal ${ }^{17,18}$. En $y$ se obtiene

16 La medida binomial se obtiene si una barra uniforme es dividida iterativamente en dos piezas de diferente tamaño, que a su vez se distribuyen uniformemente sobre un soporte geométrico, en cada etapa del proceso (Feder, 1988).

17 El término multifractal indica que en cada capa de la medida $d x$ existe un conjunto de puntos con diferente dimensión fractal (Mandelbrot, 1989). 18 Este resultado se logra cuando el parámetro $p$ adquiere un valor igual a una medida que dependerá del valor del parámetro $z^{19}$. Para la aproximación FMF, la medida encontrada en $y$ es denominada la medida derivada $d y$. Como puede apreciarse en la Figura 7, la medida derivada guarda una amplia semejanza con los patrones de alta resolución temporal que se observan en la precipitación.

Para generalizar la idea atrás del mecanismo de construcción de las medidas derivadas $(d y)$ con la aproximación FMF, nos apoyaremos en el concepto de proyección (Puente,

0.7, el cual determina la probabilidad de emplear el primer mapa afín en el proceso iterativo de construcción de la función de interpolación.

19 Para valores de $z \rightarrow 0$ se obtendrá una medida $d y$ uniforme y para valores de $z \rightarrow 1$ la medida $d y$ se parecerá a una función Gaussiana (Puente, 1996). 
1996). Este concepto establece que las medidas derivadas (dy) se construyen mediante la proyección o el mapeo de las medidas multifractales que se soportan sobre las funciones de interpolación fractal y que al ser iluminadas desde un ángulo fijo $\theta$, se pueden obtener patrones altamente intermitentes y semejantes a aquellos que son observados en geofísica (e.g. series de tiempo de precipitación). En la Figura 4S se ilustra el concepto de proyección a través de una representación tridimensional de la medidas multifractales, las cuales se observan que están soportadas en la trayectoria de una función de interpolación fractal. En el recuadro inferior de la Figura 4S, se muestran la construcción geométrica de la tormenta de Boston del 15 octubre de 1980, la cual fue estimada por Puente \& Obregón (1996) y que aquí se replica con el propósito ilustrativo de nuestras ideas.

Otras aplicaciones más recientes en el estudio de la precipitación, como son los desarrollados por Huang, Puente \& Cortis (2013) y Peñaranda (2008), denotan que los patrones obtenidos desde la aproximación FMF son bastante semejantes a los patrones simulados, conservando así, tanto la estructura estadística y multifractal de los observables. No obstante, esta aproximación sólo puede representar ventanas temporales y no la evolución temporal de los procesos de precipitación. Puente (1996) sugiere que una aproximación dinámica FMF podría llevarse a cabo si se estudiase la evolución de los parámetros, sin embargo, aún existen dificultades en la solución del problema inverso ${ }^{20}$. Huang, et al. (2013) determinaron que no existe una única codificación para un conjunto de datos observados ${ }^{21}$ y por tanto, una aproximación dinámica como la sugerida por Puente (1996) no sería factible.

Es importante destacar, que existen otros grupos de modelos que también se apoyan en la fenomenología multifractal, como son los modelos de descomposición de onditas introducido por Perica \& Foufoula-Georgiou (1996a) y los modelos de cascadas continuas anisotrópicas propuestos por Tessier, Lovejoy \& Schertzer (1993) y Lovejoy \& Schertzer (1995). Todos estos modelos intentan preservar las propiedades multifractales del campo de precipitación, apoyándose en una formulación conceptual que les permita comprender el mecanismo del escalamiento estadístico encontrado en las observaciones. Sin embargo, aún después de encontrar ajustes adecuados en los resultados de los modelos, no se ha logrado conectar la naturaleza física de los procesos de precipitación con la fenomenología multifractal empleada; por lo cual la evolución de los modelos fractales y/o multifractales, siguen generando nuevos interrogantes sobre la validez de las hipótesis incluidas en los modelos y el enlace con la realidad física que se expone en la precipitación.

20 Aquí se define el problema inverso como el proceso sistemático para determinar los parámetros del modelo a partir de un patrón observado.

21 i.e. Existen varios grupos de parámetros que pueden representar un mismo patrón.

\subsection{Modelos de base física}

Desde la base conceptual de los modelos que hasta aquí se han mencionado, es claro que no han considerado, en principio, las bases físicas de los procesos de precipitación, sin embargo otras investigaciones como las realizadas por Nordstrom \& Gupta (2003); Marzan, Schertzer \& Lovejoy. (1996); Perica \& Foufoula-Georgiou (1996b), han hecho esfuerzos por definir relaciones teóricas entre la física y la formulación matemática de los modelos descriptivos de la estructura espacio - temporal de la precipitación. El objeto de estos trabajos es, por supuesto, avanzar en la explicación física de la precipitación, pero partiendo desde un principio de parsimonia y no buscando aumentar el número de parametrizaciones de los modelos empleados en meteorología. Al respecto, ¿cómo debe ser entonces esta formulación física?¿Desde qué teoría matemática, de las aquí presentadas, debemos emprender tal formulación? Por seguro, sabemos que la precipitación guarda una estructura estadística que puede ser representada correctamente desde la teoría multifractal, sin embargo, emerge una nueva pregunta: ¿cuál es la base física de la multifractalidad de la precipitación?

Marzan, et al. (1996) proponen un modelo espacio temporal basado en la teoría de escalamiento dinámico, la cual explica la física del campo de precipitación a través de la conservación de las propiedades de simetría del ambiente turbulento en el cual se desarrolla la precipitación. En este modelo, Marzan, et al. (1996) presumen que la lluvia es un escalar pasivo que debe preservar las mismas propiedades de escalamiento de la turbulencia atmosférica. Sin embargo, Marzan, et al. (1996) encuentran que estas propiedades no se preservan. En la turbulencia no-homogénea el exponente de escalamiento anisotrópico $H$ es del orden de $1 / 3$, pero para el campo de precipitación este valor es del orden de 1/2. A pesar de esta inconsistencia, el escalamiento anisotrópico permite identificar una ruptura en la simetría del eje temporal, la cual ayuda a distinguir el pasado del futuro. Así, es factible la construcción de un modelo que describa la evolución espacio - temporal del campo de precipitación apoyado en la conceptualización de cascada multiplicativa. Algunas aplicaciones de este nuevo modelo pueden ser encontrados en Lovejoy \& Schertzer (2013).

Desde el marco de la teoría de escalamiento dinámico, Perica \& Foufoula-Georgiou (1996) formulan una relación predictiva entre las características estadísticas de la precipitación y los parámetros meteorológicos de las tormentas, postulando la existencia de una conexión empírica entre los parámetros de escalamiento y los índices termodinámicos que describen el ambiente atmosférico de las tormentas. Ellos sugieren que los parámetros de escalamiento de las observaciones de precipitación dependen de la intensidad de la inestabilidad convectiva, es decir, entre mayor sea la inestabilidad convectiva en el área en que se desarrolla la tormenta, se encontrarán valores más altos en los parámetros 
de escalamiento. Otro hallazgos encontrado por Perica \& Foufoula-Georgiou (1996), es la isotropía de los parámetros de escalamiento. Esto último sugiere que las propiedades de escalamiento no dependen de la dirección y reducen el nivel de complejidad de los modelos de precipitación.

Entre los modelos de base física y construidos sobre ecuaciones dinámicas, se encuentra el modelo introducido por Nordstrom \& Gupta (2003). Este modelo es construido para estudiar la lluvia convectiva tropical sobre el océano, independiente de consideraciones estadísticas y se interesa en entender el origen físico del escalamiento estadístico de las observaciones de precipitación. La fenomenología física del modelo se apoya en la conceptualización sugerida por Mapes (2000), la cual considera que la estructura vertical de la atmósfera es excitada por procesos de calentamiento convectivo. Bajo esta fenomenología, la intensidad de la convección profunda es modulada por la energía potencial convectiva disponible (CAPE) y la frecuencia de ocurrencia es modulada por la relación entre la inhibición convectiva (CIN) y la energía detonante (K). Los avances que se han efectuado en el estudio de este modelo, han permitido evidenciar que uno de los parámetros asociados a la CIN, puede adquirir un valor crítico que conduce a la estructura multifractal del campo de precipitaciones. Basado en estos avances, este modelo resulta promisorio para dar respuesta a las formulaciones que se enunciaron al principio de esta sección. Es importante resaltar que la característica del modelo que define una condición de criticalidad, es semejante a los fenómenos no-lineales que son encontrados en mecánica estadística y que han sido abordados desde la teoría de fenómenos críticos.

En la literatura técnica podemos encontrar otros modelos, asociados a los procesos de precipitación, que describen fenómenos de criticalidad auto-organizada. Uno de estos modelos es el introducido por Peter \& Neeling (2009, 2006), quienes sugieren que la cantidad de vapor de agua en la columna integral juega un papel de parámetro de ajuste en el desarrollo de la convección. Cuando este parámetro de ajuste alcanza un valor crítico, se presenta una transición a la precipitación fuerte. Esta transición induce a que Peter \& Neeling $(2009,2006)$ presuman que se está tratando con un proceso físico que sigue las reglas de los sistemas de criticalidad auto-organizada. En oposición a lo expreso, Muller, Back, O’Gorman \& Emanuel (2009) consideran que la evidencia empírica de criticalidad auto-organizada encontrada por Peter \& Neeling (2006) es esencialmente circunstancial, considerando que no existe un mecanismo físico que lo describa y además existen fuertes limitantes de información para verificar la consistencia de la relación que se propone. Aún las discusiones en un sentido o en otro, se mantienen abiertas.

Uno de los trabajos recientes que busca explicar la influencia de la humedad en la convección y que conecta algunas de las ideas previamente introducidas por Peter
\& Neeling $(2009,2006)$, fue desarrollado por Craig \& Mack (2013). En este trabajo se introduce un modelo de balance de humedad para la troposfera libre en el cual la fenomenología física se apoya en tres procesos: i) un proceso de secado por subsidencia, ii) un proceso de humedecimiento convectivo, y iii) un proceso de mezcla horizontal turbulento. La construcción conceptual que realiza Craig \& Mack (2013), permiten ver que la tropósfera libre puede ser representada a través de un sistema bi-estable ${ }^{22}$ en el cual los procesos de retroalimentación conducen a que las regiones secas sean más secas y las regiones húmedas sean más húmedas, dentro del campo de humedad. Craig \& Mack (2013) han denominado este proceso de concentración de la humedad, bajo el nombre de segregación. Un resultado importante del trabajo de Craig \& Mack (2013), se asocia con las propiedades fractales del modelo. Ellos identifican que el proceso de segregación preserva la propiedad de autosemejanza dinámica, es decir, la escala espacial de las estructuras $^{23}$ crecen de acuerdo a una ley de potencia en el tiempo. Adicionalmente, Craig \& Mack (2013) sugieren que la auto-organización es una propiedad genérica de los sistemas bi-estables, que es representado en el modelo por un proceso de transporte espacial débil (como es la difusión), una dependencia lineal de la tasa de secado por subsidencia, una dependencia exponencial en la tasa de humedecimiento convectivo y una restricción determinada por la hipótesis de equilibrio radiativo - convectivo.

Es importante aclarar que el modelo introducido por Craig \& Mack (2013), es una aproximación para describir el campo de humedad en el cual se desarrolla la convección; por tanto, aún se desconoce el canal para que los resultados que se derivan de este modelo puedan representar el campo de precipitación. No obstante, este modelo reúne un conjunto de propiedades interesantes a resaltar: la naturaleza física en la cual se desarrolla la convección, las propiedades fractales del proceso de segregación y una estructura conceptual que se enmarca en la teoría de los fenómenos críticos. Desde la base conceptual introducida por Craig \& Mack (2013), se enmarca una nueva ruta en el estudio de la precipitación, la cual podrá ser abordada si se logra traducir el modelo de balance de humedad en un modelo de lluvia y si se estudian las propiedades estadísticas y multifractales del campo simulado. Pero, el reto científico, es necesario abordarlo con cautela, puesto que la respuesta del modelo deberá ser contrastada con las observaciones que estén a la escala adecuada y en la cual el proceso de segregación pueda ser evidenciado.

22 En la teoría de los fenómenos críticos, un sistema bi-estable es representado por dos estados de equilibrio. Estos dos estados de equilibrio son dados por que la función de energía libre se compone de dos puntos críticos que representan dos estados de mínima energía.

23 En este contexto, las estructuras representan las regiones en las cuales se concentra la humedad o las regiones que se hacen más secas. 


\section{Conclusiones}

Cuatro aspectos esenciales han sido discutidos e ilustrados a lo largo del artículo: i) La importancia práctica y científica de avanzar en el conocimiento de la precipitación. ii) La complejidad de la estructura espacio - temporal de la precipitación mediante ilustraciones en el caso colombiano, que exhibe gran variabilidad espacial en cortas distancias, cambios importantes a escala inter-anual, anual, intraanual, diaria y de tormenta. Aunque hay explicaciones parciales, subsisten múltiples preguntas. A escala mayor en el espacio - tiempo puede haber algún grado de suavización de la gran variabilidad de la escala fina, pero no desaparece la variabilidad, y tampoco las preguntas. Por ejemplo, el efecto del cambio climático tiene niveles no despreciables de incertidumbre, no sólo por el signo y la magnitud del cambio y su estructura espacial, sino también por su estructura espacio - temporal. Puede darse el caso de un sitio donde se presenta una tendencia decreciente en la precipitación a escala multi-anual y puede estar sometido a tormentas intensas más frecuentes. iii) Se describen mediante modelos representativos, los principales enfoques que se han usado para explicar la precipitación y se hace énfasis en sus limitaciones. iv) Se discuten las posibles rutas para futuras investigaciones en el estudio de la precipitación.

Las formulaciones conceptuales que se han enunciado en este artículo, muchas de ellas con un carácter puramente matemático, presentan resultados que parecen adecuados para representar el campo de precipitación; sin embargo, debe tenerse presente que en los ajustes de los modelos, solamente logran representar algunas características estadísticas o geométricas de los patrones observados de precipitación a la escala usada para ajustar el modelo, pero su falta de integralidad con la física del proceso, no garantizan la universalidad de sus teorías. Como consecuencia de lo anteriormente indicado, las preguntas científicas sobre la descripción espacio - temporal de la precipitación se encuentran abiertas, lo que trae consecuencias para las aplicaciones en el contexto pragmático de la ingeniería. En la medida en que los últimos resultados teóricos de las ciencias físicas y matemáticas sean acoplados como herramientas de estudio y entendimiento de los procesos hidrológicos, más acertadas e integradas serán las teorías dinámicas de la precipitación. Ejemplos de ello se puede evidenciar en los trabajos de Nordstrom \& Gupta (2003), Peters \& Neelin (2006, 2009) y Craig \& Mack (2009), quienes vislumbran el desarrollo de un fenómeno crítico en los procesos de precipitación que ocurre durante las transiciones de fase en la atmósfera, y aunque estos trabajos no están enmarcados directamente a explicar la naturaleza física de la multifractalidad, creemos que en estas conceptualizaciones puede estar la clave para este fin. Un avance dentro de este marco permitirá comprender mejor la variabilidad del campo de precipitación en todas sus escalas de desarrollo y aún más, las relaciones que puedan existir entre todas las escalas. Lo último, tendría un impacto importante para los estudios hidrológicos que se realizan en regiones con información escasa, puesto que se tendría la total claridad para establecer un protocolo de desagregación de la información e inclusive, para optimizar las técnicas de diseño en ingeniería hidráulica.

A nuestro juicio, los trabajos futuros deben estar enmarcados a establecer los límites funcionales de todas las teorías, no a lograr mejores ajustes de los modelos. Se debe intentar aclarar cuales de ellos verdaderamente proporcionan una explicación válida y universal sobre la dinámica espacio temporal de la precipitación. Unos primeros pasos en ese sentido, estarán en: i) demarcar relaciones concluyentes entre la fenomenología de cascadas multifractales y las ecuaciones dinámicas fundamentales de la atmósfera, ii) verificar si el origen del escalamiento multifractal de la precipitación tropical proviene como respuesta a un fenómeno critico termodinámico y iii) a la luz de los avances en el entendimiento de la dinámica de los procesos de precipitación, identificar su eficacia para las aplicaciones. Estas aplicaciones, no sólo consisten en la re-formulación de procedimientos convencionales para la toma de decisiones en ingeniería, también comprende la conjugación de nuevas herramientas para estudiar escenarios de cambio climático, los cuales finalmente determinan el porvenir social y económico de la humanidad.

\section{Información suplementaria}

Figura 1S. Campos mensuales de precipitación medios mensuales (en mm/mes) para Colombia durante el evento La Niña de 1999 - 2000, los cuales fueron calculados por Hurtado (2009) a partir de 2.270 estaciones pluviográficas y las bases de datos del NCEP, GPCP, TRMM, GOES. La interpolación usa una modificación del modelo PRISM (Daly, et al., 1994, 2008) descrita en Hurtado (2009) y Hurtado \& Mesa (2014).

Figura 2S. (arriba) Series de tiempo de valores de intensidad media de precipitación (en mm/hora) del año 1999 para las escalas de agregación temporal $\mathrm{T}=30 \mathrm{~min}$, 3h y $6 \mathrm{~h}$ medidos en la estación pluviográfica Bosa Barreno (Bogotá) y (abajo) la relación entre la intensidad máxima y la escala de agregación para las series de tiempo observadas en Bosa Barreno en el año 1999 y las que resultan durante el proceso de agregación temporal de una realización con el modelo de pulsos rectangulares de Poisson a la escala $\mathrm{T}=30 \mathrm{~min}$. Por la apariencia que describen las series de tiempo de intensidad media de precipitación (en el recuadro superior), su comportamiento puede ser asociado a un proceso aleatorio; razón por la cual, muchos investigadores han empleado los modelos estocásticos para la descripción de la precipitación (e.g. Rodríguez-Iturbe, (1986) y Rodríguez-Iturbe, et al. (1987, 
1984)). No obstante, los modelos estocásticos no logran capturar toda la estructura estadística y geométrica de las observaciones, como se describe en el recuadro inferior de la figura, donde las intensidades máximas de las series observadas despliegan un decaimiento exponencial con la escala, más rápido que el presente en la simulación estocástica.

Figura 3S. Serie de tiempo de valores totales de precipitación (en mm) del periodo comprendido por los años 1995 a 1999 para la escala de agregación temporal T = 30 min, medidos en la estación pluviográfica Bosa Barreno (Bogotá). Esta serie de tiempo será empleada en el artículo para la estimación de los momentos estadísticos y la función de estructura, los cuales se ilustran en la Figura 6.

Figura 4S. La forma geométrica que adquiere la medida derivada de la aproximación FMF puede ser comprendida si se asume que una medida multifractal, la cual es sostenida sobre las trayectorias que definen una función de interpolación fractal, es proyectada como iluminaciones sobre un plano perpendicular al eje $x$ e $y, y$ orientado desde cualquier ángulo sobre el eje $d x$.

\section{Conflicto de intereses}

Los autores declaran que no tienen ningún conflicto de intereses.

\section{Bibliografía}

Adler, R., Gu, G., \& Huffman, G. (2011). Estimating climatological bias errors for the global precipitation climatology project GPCP. Journal of Apply Meteorology and Climatology, 51 (1): 84-99.

Barnsley, M. (1993). Fractals Everywhere. (2 ed.). San Diego, California: Academic Press, Inc.

Bhattacharya, R. \& Waymire, E. (2009). Stochastic processes with applications. New York: Siam.

Bookhagen, B. \& Strecker, M. (2008). Orographic barriers, highresolution TRMM rainfall, and relief variations along the eastern Andes. Geophysical Research Letters, 35 (6).

Craig, G. \& Mack, J. (2013). A coarsening model for selforganization of tropical convection. 118 (16): 8761-8769.

Daly, C., Neilson, R. \& Phillips, D. (1994). A statisticaltopographic model for mapping climatological precipitation over mountainous terrain. Journal of Applied Meteorology, 33 (2): 140-158.

Daly, C., Halbleib, M., Smith, J., Gibson, W., Doggett, M., Taylor, G., Curtis, J. \& Pasteris, P. (2008). Physiographically sensitive mapping of climatological temperature and precipitation across the conterminous United States. International Journal of Climatology, 28 (15): 2031-2064.

Feder, J. (1988). Fractals (Physics of solids and liquids). New York: Springer Science + Business Media.

Feller, W. (1967). An introduction to probability theory and its applications. Volume I (3 ed.). New York: John Wiley \& Sons, Inc.

Gómez, J. \& Poveda, G. (2008) Estimación del espectro multifractal para series de precipitación horaria en los Andes tropicales de Colombia. Revista de la Academia Colombiana de ciencias exactas, físicas y naturales, 32 (125): 483-502.

Gupta, V. \& Waymire, E. (1993). A statistical analysis of mesoscale rainfall as a random cascade. Journal of American Meteorological Society. 32: 251-267.
Hipel, K. \& McLeod, A.I. McLeod. (1994). Time series modelling of water resources and environmental systems. Amsterdam: Elseviewer.

Hoyos, C. \& Webster, P. (2006). Estructura espacio - temporal de la variabilidad intra-estacional en el trópico. Avances en Recursos Hidráulicos. 14 (3): 3-24.

Huang, H, Puente, C. \& Cortis, A. (2013). Geometric harnessing of precipitation records: reexamining four storms from Iowa City. Stochastic Environmental Research and Risk Assessment, 27 (4): 955-968.

Hurtado, A. (2009). Estimación de los campos mensuales de precipitación en el territorio colombiano. (Tesis de Maestría). Universidad Nacional de Colombia, Sede Medellín, Facultad de Minas. Medellín.

Hurtado, A. \& Mesa, Ó. (2014). Reconstrucción de los campos de precipitación mensual en Colombia. Revista Dyna. 81 (186): 251-258.

IPCC. (2013). Climate Change 2013: The Physical Science Basis. Fifth Assessment Report of the Intergovernmental Panel on Climate Change (AR5). New York: Cambridge University Press.

Lovejoy, S. \& Schertzer, D. (1995). Multifractals and rain. En Zbigniew W. Kundzewicz (ed.). New Uncertainty Concepts in Hydrology and Water Resources, (pp.61-103). Cambridge: Cambridge University Press.

Lovejoy, S \& Schertzer, D. (2013). The weather and climate: emergent laws and multifractal cascades. New York: Cambridge University Press.

Mandelbrot, B. (1982). The fractal geometry of nature. New York: W.H. Freeman and Company.

Mandelbrot, B. (1989). Multifractal measures, especially for the geophysicist. Pure and Applied Geophysics, 131 (12): 5-42.

Mapes, B. (2000). Convective inhibition, subgrid-scale triggering energy, and stratiform instability in a toy tropical wave model. Journal of Atmospheric Sciences, 57 (10): 15151535.

Marzan, D., Schertzer, D. \& Lovejoy, S. (1996). Causal space - time multifractal processes: predictability and forecasting of rain fields. Journal of Geophysical Research. 101:D21(26): 333-346. 
Mejía, J. (2002). Ciclo diurno de la lluvia y ambientes atmosféricos en sistemas convectivos de mesoescala sobre Colombia y el este del océano pacifico usando datos de la TRMMy del reanálisis del NCEP/NCAR (Tesis de Maestría). Universidad Nacional de Colombia, Sede Medellín, Facultad de Minas. Medellín.

Mesa, Ó., Poveda, G., \& Carvajal, L. (1997). Introducción al clima de Colombia. Medellín: Editorial Universidad Nacional de Colombia, Sede Medellín, Facultad de Minas.

Mesa, Ó. (2007). ¿Adónde va caer este globo? Medellín: Editorial Universidad Nacional de Colombia, Sede Medellín, Facultad de Minas.

Muller, C., Back, L., O’Gorman, P. \& Emanuel, K. (2009). A model for the relationship between tropical precipitation and column water vapor. Geophysical Research Letters, 36 (16).

National Research Council. (1991). Opportunities in the Hydrologic Sciences. Washington: National Academy Press. Committee on opportunities in the hydrologic sciences, water science \& technology board, commission on geosciences, environment, and resources edition.

Nordstrom, K. \& Gupta, V. (2003). Scaling statistics in a critical, nonlinear physical model of tropical oceanic rainfall. Nonlinear Processes Nonlinear Processes in Geophysics, 10: $531-543$.

Over, T. \& Gupta, V. (1994). Statistical analysis of mesoscale rainfall: dependence of a random cascade generator on large-scale forcing. Journal of Hydrology, 33: 1526-1542.

Over, T. (1995). Modelling space - time mesoscale rainfall using random cascades (Doctoral Dissertation. Ph.D. in Geophysics). University of Colorado. Boulder, Colorado.

Over, T. \& Gupta, V. (1996). A space-time theory of mesoscale rainfall using random cascades. Journal of Geophysical Research, 101 (26): 319-331.

Paschalis, A., Molnar, P., Fatichi, S. and Burlando, P. (2013) A stochastic model for high-resolution space - time precipitation simulation. Water Resources Research, 49 (12): 8400-8417.

Peñaranda, V. (2008). Representación geométrica de registros de precipitación puntual en Bogotá con el modelo Fractal Multifractal (Tesis de Maestría), Universidad Nacional de Colombia, Sede Bogotá, Facultad de Ingeniería. Bogotá.

Perica, S. \& Foufoula-Georgiou, E. (1996a). A Model for multiscale disaggregation of spatial rainfall based on coupling meteorological and scaling descriptions, Journal of Geophysical Research, 101 (D21), 26: 347-26,361.

Perica, S. \& Foufoula-Georgiou, E. (1996b). Linkage of scaling and thermodynamic parameters of rainfall: results from midlatitude mesoscale convective systems. Journal of Geophysical Research, 101 (D3): 7431-7448.

Peters, O. \& Neelin, J. (2006). Critical phenomena in atmospheric precipitation. Nature Physics, 2: 393-396.

Peters, O. \& Neelin, J. (2009). Atmospheric convection as a continuous phase transition: Further evidence. International Journal of Modern Physics B, 23 (28-29): 5453-5465.
Poveda, G. and Mesa, Ó. (1999). La corriente de chorro superficial del oeste (del Chocó) y otras dos corrientes de chorro atmosféricas sobre Colombia: Climatología y variabilidad durante las fases de ENSO. Revista Academia Colombiana de Ciencias, 23 (89): 514-528.

Poveda, G., Mesa, Ó., Agudelo, P., Álvarez, J., Arias, P., Moreno, J., Salazar, L. \& Toro, V. (2002a). Influencia del ENSO, oscilación Madden - Julian, ondas del este, huracanes y fases de la luna en el ciclo diurno de la precipitación en los Andes tropicales de Colombia. Revista Meteorología Colombiana, 5: 3-12.

Poveda, G., Mesa, Ó., Agudelo, P., Álvarez, L., Arias, P., Moreno, H., Salazar, L., Toro, V., Vieira, S., Jaramillo, A. \& Guzmán, O. (2002b). Diagnóstico del ciclo diurno de la precipitación en los Andes tropicales. Revista Meteorología Colombiana, 5: 23-30.

Poveda, G., Mesa, Ó., Toro, V., Agudelo, P., Álvarez, J., Arias, P., Moreno, H. \& Salazar, L. (2002c). Diagnóstico del ciclo anual y efectos del ENSO sobre la intensidad máxima de lluvias de duración entre 1 y 24 horas en los andes tropicales. Revista Meteorología Colombiana, 5: 67-74.

Poveda, G. (2004). La hidroclimatología de Colombia: una síntesis desde la escala inter-decadal hasta la escala diurna. Revista Academia Colombiana de Ciencias, 28 (107): 201-222.

Poveda, G., Mesa, Ó., Salazar, L., Arias, P., Moreno, H., Vieira, S., Agudelo, P., Toro, V. \& Álvarez, J. (2005). The diurnal cycle of precipitation in the tropical Andes of Colombia. Monthly Weather Review, 133: 228-240.

Puente, C. (1996). A new approach to hydrologic modeling: derived distribution revisited. Journal of Hydrology, 187: 65-80.

Puente, C. \& Obregón, C. (1996). A deterministic geometric representation of temporal rainfall: results for a storm in Boston. Water Resources Research, 32: 2825-2839.

Rodríguez-Iturbe, I., Gupta, V. \& Waymire, E. (1984). Scale considerations in the modeling of temporal rainfall. Water Resources Research, 20 (11): 1611-1619.

Rodríguez-Iturbe, I. (1986). Scale of fluctuation of rainfall models. Journal of Water Resources Research, 22 (9S): 15S-37S.

Rodríguez-Iturbe, I., de Power, F. \& Valdés, J. (1987). Rectangular pulses point process models for rainfall: Analysis of empirical data. Journal of Geophysical Research: Atmosphere, 92 (D8): 9645-9656.

Rodríguez-Iturbe, I. Exploring complexity in the structure of rainfall. (1991). Advances in Water Resources, 14 (4): 162-167.

Schertzer, D. \& Lovejoy, S. (2011). Multifractals, generalized scale invariance and complexity in geophysics. Bifurcation and Chaos, 21 (12): 3417-3456.

Tessier, Y., Lovejoy, S. \& Schertzer, D. (1993). Universal multifractals: theory and observations for rain and clouds. Journal of Apply Meteorology, 32: 223-250. 